\title{
Bimodal Interactions in the Superior Colliculus of the Behaving Cat
}

\author{
Luis C. Populin ${ }^{1,3}$ and Tom C. T. Yin ${ }^{2,3}$ \\ ${ }^{1}$ Department of Anatomy, ${ }^{2}$ Department of Physiology, and ${ }^{3}$ Neurosciences Training Program, University of Wisconsin- \\ Madison, Madison, Wisconsin 53706
}

Bimodal enhancement, a form of nonlinear summation of physiological responses from two sensory modalities, has been demonstrated in the intermediate layers of the superior colliculus (SCi) and is thought to be a manifestation of a neural mechanism underlying behavioral facilitation to such stimuli. Most physiological studies, however, have been performed in anesthetized animals. We tested for bimodal enhancement in the SCi of behaving cats trained to orient to acoustic, visual, and bimodal stimuli. Surprisingly, we never observed the large enhanced responses reported in anesthetized animals, even

Interactions between sensory modalities influence our perceptions and behaviors: they may affect shifts of attention (Driver and Spence, 1998), sensory discrimination (Butter et al., 1989; Farah et al., 1989), or reaction times (Frens et al., 1995; Schröger and Widman, 1998; Taylor et al., 1999). Although we have learned much about the psychophysics of such interactions, their underlying physiological mechanisms are less clear, because most physiological studies have been performed in anesthetized preparations.

The superior colliculus (SC) has served as a model for studying multisensory integration (Stein and Meredith, 1993). Its intermediate layers (SCi) receive ascending and descending sensory inputs (Huerta and Harting, 1984), where they form representations of auditory (Gordon, 1973; King and Palmer, 1983; Wise and Irvine, 1983; Middlebrooks and Knudsen, 1984; Hirsch et al., 1985) and visual (Stein et al., 1976; Finlay et al., 1978) space and body surface (Cynader and Berman, 1972; Meredith et al., 1991). There are also motor maps of eye (Robinson, 1972; Schiller and Stryker, 1972; Guitton et al., 1980) and pinna (Stein and Clamann, 1981) movements. In anesthetized animals, such maps are in spatial register (Gordon, 1973; Stein et al., 1976; King and Palmer, 1983, 1985; Meredith and Stein, 1986a,b), which in behaving animals is thought to be actively maintained to compensate for eye movements (Jay and Sparks, 1987; Populin and Yin, 1998a). Approximately $50 \%$ of SCi cells are multisensory (Meredith and Stein, 1986b). By responding to stimuli from more than one modality and by projecting to downstream motor centers (Meredith and Stein, 1985), the SCi integrates the different sensory modalities and motor systems.

\footnotetext{
Received Oct. 25, 2001; revised Dec. 26, 2001; accepted Dec. 27, 2001.

This work was supported by National Institutes of Health Grants DC00116 and DC02840 (T.C.T.Y.) and DC03693 (L.C.P), National Science Foundation Grant IBM-9904770 (L.C.P.), the Deafness Research Foundation (L.C.P. and T.C.T.Y.), and the National Organization for Hearing Research (L.C.P.). We thank John Hudson and Dan Tollin for assistance, Ravi Kochhar and Jane Sekulski for programming help, Ray Guillery, Dan Tollin, and Dan Uhlrich for reading earlier drafts of this manuscript, and Doris Kistler for discussions about the statistics.

Correspondence should be addressed to Luis C. Populin, Department of Anatomy, 1300 University Avenue, Madison, WI 53706. E-mail: lpopulin@facstaff.wisc.edu.

Copyright (C) 2002 Society for Neuroscience $0270-6474 / 02 / 222826-09 \$ 15.00 / 0$
}

when we varied the time between presentation of the visual and acoustic stimuli and/or decreased the level of the stimuli. Using three different behavioral paradigms, we found no support for enhanced interactions between auditory and visual modalities. Prominent depressive effects were seen, however, particularly when the cats were required to fixate a visual target during presentation of an acoustic stimulus.

Key words: superior colliculus; bimodal; multisensory; enhancement; suppression; behaving cat

In anesthetized animals, SCi cells can add inputs from different sensory modalities nonlinearly, resulting in some cases in bimodal enhancement or suppression (Newman and Hartline, 1981; Meredith and Stein, 1983). By responding to bimodal stimuli with responses that exceed the larger of the unimodal responses (Meredith and Stein, 1983) or the sum of the unimodal responses (King and Palmer, 1985), the SCi is thought to increase the likelihood of behavioral responses (Stein and Meredith, 1993). King and Palmer (1985) found suppressive responses of as much as $-95 \%$ and enhancement of up to $+452 \%$ in the guinea pig, whereas Meredith and Stein $(1983,1986 b)$ showed examples from the cat that appear to be in the range of -99 to $+300 \%$ (Meredith and Stein, 1983; their definition of interaction took into account only the larger of the unimodal responses) or $20 \%$ to infinity (Meredith and Stein, 1986b).

Because the terms have been used differently by different authors, there is a need to define precisely what we mean by bimodal enhancement. In this article, we consider bimodal enhancement to be bimodal responses that exceed the sum of the individual unimodal responses, as defined by King and Palmer (1985), whereas bimodal suppression occurs when the bimodal response is less than the larger of the unimodal responses. We believe this more accurately reflects the fundamental notion than the definition used by Meredith and Stein $(1983,1986 b)$, in which enhancement is defined as a bimodal response larger than the larger of the unimodal responses. Ironically, the measure used by Meredith and Stein $(1983,1986 b)$ to measure enhancement is actually more suited to measuring suppression. Neither measure on its own can provide a quantification of both enhancement and suppression.

Although bimodal responses have been documented in behaving preparations (Peck, 1996; Populin and Yin, 1997), evidence linking behavioral facilitation to enhanced bimodal interactions (BIs) in the SCi is indirect at best (Stein et al., 1989). In fact, enhanced BIs, of the magnitude demonstrated in anesthetized animals, have not been reported in behaving preparations (Peck, 1996; Frens and Van Opstal, 1998) (but see Wallace et al., 1998), yet they are cited in the literature as a mechanism underlying 
bimodal behavioral facilitation (Driver and Spence, 1998; Frens and Van Opstal, 1998). The present experiments were performed to test for the presence of enhanced BIs in the SCi of behaving cats and to quantify their magnitude.

\section{MATERIALS AND METHODS}

Subjects and surgery. A detailed description of the behavioral training methods has been published previously (Populin and Yin, 1998b). Briefly, experiments were performed in four domestic cats trained to orient to acoustic, visual, and bimodal stimuli. Each animal underwent two sterile surgical procedures: the first to implant eye coils (Judge et al., 1980) and a head post and the second to implant a recording cylinder to access the SC with microelectrodes. All experimental procedures were approved by the University of Wisconsin Animal Care Committee and were in accordance with the National Institutes of Health Guide for the Care and Use of Laboratory Animals.

Experimental setup, presentation of stimuli, and eye movement recording. Experiments were performed in a single-walled sound-attenuating recording chamber with the interior and major pieces of equipment covered with reticulated foam to minimize acoustic reflections. The acoustic stimulus was a noise burst $(0.1-25 \mathrm{kHz}$ with $\pm 7 \mathrm{msec}$ linear rise-fall) presented through Radio Shack super tweeters (model 40-1310A) modified to transduce low frequencies. Unless otherwise noted, the level of the acoustic stimuli was $\sim 20 \mathrm{~dB}$ above the threshold of each of the cells studied. The visual stimuli were stationary red light-emitting diodes (LEDs) placed at the center of each speaker. Sixteen speaker-LED ensembles were used to present the stimuli, positioned on a cross pattern along the horizontal and vertical planes in the frontal hemifield; the minimum separation between speakers was $9^{\circ}$. Eye movements were measured with the magnetic search coil technique (Robinson, 1963). The output of the search coil system (CNC Engineering, Seattle, WA) was sampled at $500 \mathrm{~Hz}$ with an analog-to-digital converter. Both stimulus presentation and data collection were controlled by custom software running on a MicroVAX-2 computer (Digital Equipment Co., Maynard, MA).

Behavioral training, experimental tasks, and electrophysiology. In all experiments, the heads of the animals were restrained. The cats were trained to perform several experimental tasks (fixations, standard and delayed saccades, and sensory probes) (Populin and Yin, 1998b). A typical experimental session consisted of a series of trials of the various tasks involving any one of 16 targets positioned along the horizontal and vertical planes in the frontal hemifield using acoustic, visual, or bimodal stimuli. The task type, target location, and stimulus type were randomly intermingled so that the cat could not predict the task or parameters of the next trial. The physiological recordings presented below were obtained with the fixation and sensory probe tasks; other tasks were included to maintain the randomness of the tasks.

In the fixation task (Fig. 1A), the trial began with the appearance of the stimulus, and the cat was required to look to the source of the stimulus and to maintain fixation until it was extinguished to obtain a food reward. Typically, the cat would be looking downward toward the feed tube while getting the reward from the previous trial. As the intertrial interval was ending, the cat would usually bring its eyes up near the center of gaze in apparent anticipation of the next trial. The duration of the fixation stimulus was varied randomly $(1000-1500 \mathrm{msec})$ for the different targets. On any given trial, the stimulus could be an acoustic, visual, or bimodal one, as illustrated in Figure $1 A$. The sensory probe task (Fig. $1 B$ ) was used to control the position of the cat's eyes during the trial. It required the cat to fixate the LED located at the primary position (stimulus 1 in Fig. $1 B$ ). After a delay of $300-700 \mathrm{msec}$, a visual, acoustic, or bimodal stimulus was presented within the receptive field of the cell while the cat continued to fixate the LED; a reward was delivered if the animal maintained fixation for the duration of the trial.

Possible differences between the responses in the fixation and sensory probe tasks may be attributable to the position of the eyes, the presence of a visual fixation stimulus, and the behavioral relevance of the stimulus. Because the responses in SC cells usually had a strong onset component, in both tasks they occurred while the cat's eyes were near the center of gaze. In the fixation trials, however, the eye position at the time the stimulus was turned on was not under direct experimental control, whereas in the sensory probe trials, an LED was present at the center of gaze. In fixation trials, the stimulus was the target for a saccade, whereas it was behaviorally irrelevant in the sensory probe trials. However, we
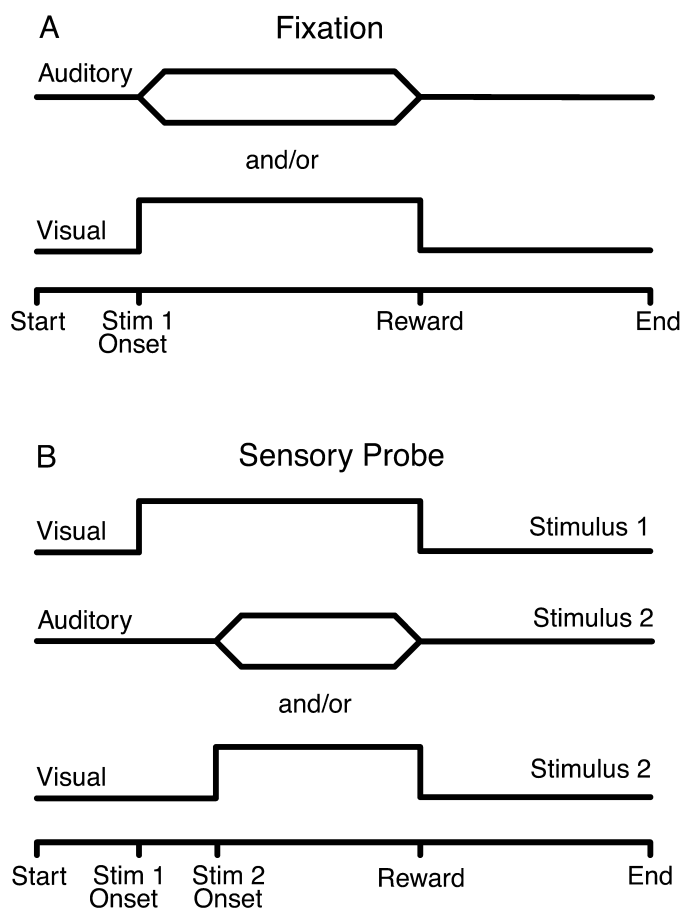

Figure 1. Schematic representation of the experimental tasks. Fixation task $(A)$ consisted of a visual, acoustic, or bimodal (visual and acoustic) stimulus. To receive a reward, the cat was expected to look at the source of the stimulus and to maintain fixation until it was extinguished. The sensory probe task $(B)$ consisted of a visual fixation stimulus (Stimulus 1 ) presented at the primary position. The cat was required to look at this target, during the presentation of which a second stimulus (Stimulus 2), which could be visual, acoustic, or bimodal, was presented at an eccentric position. The cat earned a reward by maintaining fixation on stimulus 1 for the duration of the trial.

believe this difference in behavioral relevance is mitigated by the fact that the sensory probe task is a variation of the delayed saccade task we used for training (Populin and Yin, 1998b), in which the animal had to make a saccade to a target cued by the offset of the fixation LED after a short delay (300-500 msec) after target onset. The first part of a sensory probe trial is identical to a delayed-saccade trial; thus, we expected the animals to treat a probe stimulus as a potential target for a saccade. Thus, we believe that response differences in the fixation and sensory probe tasks are not likely to be attributable to the position of the eyes or the behavioral relevance but rather to the presence of the visual fixation stimulus or the act of fixation itself.

We assumed that when the eyes of the subject were at the primary position, the auditory and visual maps in the SC were in spatial register. Standard extracellular recording techniques were used to study the responses of single cells while the cats were actively performing the behavioral tasks.

Data analysis. Two different metrics have been used to evaluate and compare the bimodal response with the unimodal responses in bimodal $\mathrm{SC}$ cells. To test for bimodal enhancement, we used the percentage of BI of King and Palmer (1985):

$$
B I=[(B i-V-A) /(V+A)] \times 100,
$$

where $B i$ is bimodal, $V$ is visual, and $A$ is auditory response in number of spikes computed over a $200 \mathrm{msec}$ window starting $5 \mathrm{msec}$ after stimulus presentation; most responses, particularly those obtained with the sensory probe task, were transient and had expired before $200 \mathrm{msec}$. By this measure, bimodal enhancement would result in $\mathrm{BI}>0$. The mean BI obtained from each of the experimental conditions was tested to determine whether it differed significantly from 0 using a $t$ test. The metric adopted by Meredith and Stein $(1983,1986 \mathrm{~b})$ only compares the bimodal response with the larger of the unimodal responses, while ignoring the weaker unimodal response. We believe that this metric is not appropriate 
for testing for enhancement but is suitable for testing for suppression, so we will refer to it as the percentage of bimodal suppression (BS):

$$
B S=\left[\left(B i-U n i_{\max }\right) / U n i_{\max }\right] \times 100,
$$

where $B i$ is bimodal response, and $U n i_{\max }$ is the larger of the unimodal responses. Bimodal suppression occurs when $\mathrm{BS}<0$. Bimodal responses less than the sum of the unimodal but larger than $U n i_{\max }$ are considered occluded.

\section{RESULTS}

\section{Bls in SCi cells}

We recorded from 98 single cells in the SCi of four behaving cats. All but six cells, which did not respond to visual stimuli, were bimodal. Typical oculomotor behavior of the cat orienting to visual, acoustic, and bimodal targets in the fixation task and the responses of a single cell are illustrated in Figure 2. The targets were located at $0^{\circ},-23^{\circ}$. Only the vertical component of the eye movements is shown for simplicity; all traces are plotted synchronized at $0 \mathrm{msec}$ to the time at which the stimuli were turned on. In most trials, the eyes of the cat were near the straight-ahead position at the time of stimulus presentation. The eye movements (Fig. 2, top) to the bimodal target were more stereotyped and consistent than those to visual and acoustic targets, and as expected, they had shorter latency (visual: $188.3 \mathrm{msec}, \mathrm{SD} \pm 48.9$; auditory: $213.0 \mathrm{msec}, \mathrm{SD} \pm 95.4$; bimodal: $125.7 \mathrm{msec}, \mathrm{SD} \pm 26.7$ ).

The cell, responses of which are shown in Figure 2, middle and bottom, was bimodal: it responded to the presentation of either a visual, acoustic, or bimodal stimulus placed in its receptive field as shown in Figure 2 (middle and bottom). This single cell encoded an area of space below the horizontal plane near the midline. Typically, responses to single or bimodal stimuli consisted of a transient burst followed by irregular lower-frequency activity. As in all cells in our sample, the response latency was shorter for the acoustic stimulus $(16.9 \mathrm{msec})$ than for the visual one $(68 \mathrm{msec})$. The responses to the bimodal stimuli approximated the sum of the two single-modality stimuli. The BI of 7.5\% indicates that the bimodal response was only slightly larger than the sum of the two single-modality responses. Note that the timing of the bursts of the single-modality responses was well preserved in the bimodal response, as evidenced by the two peaks in the bimodal response, reflecting the difference in response latencies. This cell was unusual in that it was one of the few that had a BI of $>0$. A histogram summarizing the BI values for the 80 cells studied with the fixation task is shown in Figure 3. In no case did we observe large enhanced responses resembling those reported in anesthetized animals (Meredith and Stein, 1983; King and Palmer, 1985). To the contrary, only 14 of $80(17.5 \%)$ of the responses had $\mathrm{BI}>0$, and the mean $\mathrm{BI}$ value was significantly smaller than 0 (mean $\mathrm{BI}=-19.0 \%, \mathrm{SD} 23.2 \% ; t_{(79)}=6.01, p$ $<0.05$ ). Thus, these data show no support for bimodal enhancement. Bimodal suppression was evident for a large proportion of cells: 30 of $80(37.5 \%)$ had $\mathrm{BS}<0$.

\section{Spatial register}

Spatial register among the various maps in the SC, a prominent feature of the SC of anesthetized preparations (Meredith and Stein, 1996), is thought to be a requirement for bimodal enhancement to occur (Stein and Meredith, 1993). In the fixation task, we did not control the position of the cat's eyes, although, as can be seen in Figure $2 A$, they were usually near the primary position in anticipation of the beginning of the next trial. Variations in eye position could account for the lack of bimodal enhancement if the auditory and visual maps of the SCi were not in spatial register at the time of stimulus presentation. We think that this is an unlikely explanation for our results, however, because of the large size of receptive fields of SCi cells (Stein and Meredith, 1993) and because in behaving subjects the visual and auditory maps in the $\mathrm{SC}$ are thought to be maintained in register by an active process (Jay and Sparks, 1987; Populin and Yin, 1998a). Nonetheless, to ensure that the various SC maps were indeed in spatial register, we used a sensory probe task, which kept the eyes of the cat straight ahead during the presentation of the stimulus.

The responses of a typical SCi cell to acoustic, visual, and bimodal stimuli recorded with the sensory probe task are shown in Figure 4. Both the visual and the acoustic stimuli presented alone evoked responses characterized by an initial transient burst. The responses to bimodal stimuli were smaller than the sum of

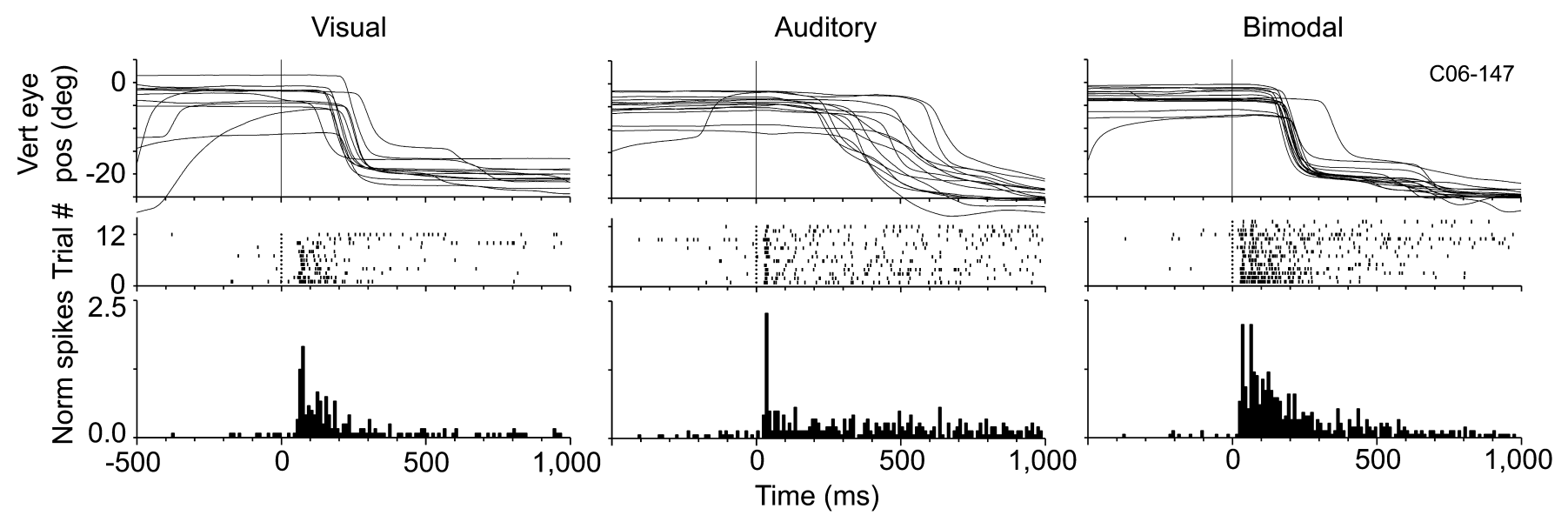

Figure 2. Responses of a bimodal cell in the SCi of a behaving cat with the fixation task. Top, Vertical component of eye movements to visual (left), acoustic (center), and bimodal (right) targets located at $\left(0^{\circ},-23^{\circ}\right)$; the horizontal component is not shown for simplicity. The visual stimulus was a stationary red LED, and the acoustic stimulus was a broadband noise burst. Single-cell responses recorded in individual trials are illustrated by the dot rasters (center) and summarized by the histograms (bottom), which are normalized by the number of trials in each condition; $10 \mathrm{msec}$ bins were used. In all dot rasters, the time of occurrence of the action potentials is plotted with a small vertical line, whereas behavioral events (e.g., stimulus onset at $0 \mathrm{msec}$ ) are denoted by small dots and are not included in the histograms. All data are plotted synchronized to the onset of the stimuli. The BI for this cell was $7.5 \%$. 


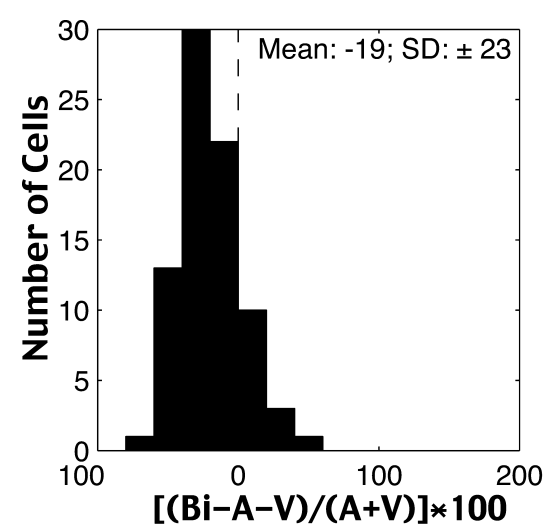

Figure 3. Summary of BI values recorded with the fixation task. Responses were computed over a $200 \mathrm{msec}$ window starting $5 \mathrm{msec}$ after stimulus presentation. The vertical broken line marks $\mathrm{BI}=0$.

the responses to the single-modality stimuli presented alone, with a BI of $-19.5 \%$. Again the response latency to the acoustic stimulus was shorter than to the visual, and the timing of the bursts of the single-modality responses was well preserved in the response to the bimodal stimuli. We studied 52 cells with the sensory probe task, 35 of which were also studied with the fixation task. The summary of the results obtained with the sensory probe task (Fig. 5 ) again showed no evidence for bimodal enhancement: only 13 of $52(25 \%)$ of the cells had BI $>0$, although in this case the $95 \%$ confidence interval $(-21 \%$ to $+1 \%)$ for the mean BI $(-9.9 \%$, SD $39)$ just barely included zero $\left(t_{(51)}=1.74, p>0.05\right)$. Bimodal suppression was seen in $38.5 \%$ (20 of 52) of the cells that had $\mathrm{BS}<0$.

\section{Effects of delaying the acoustic stimuli}

Bimodal cells in the SCi respond to visual and acoustic stimuli with different latencies, usually with a shorter latency to the acoustic stimulus, as illustrated in the responses shown in Figures 2 and 4 . For the 80 cells we studied with the fixation task, the mean first spike latency to acoustic stimuli (17.7 msec; SD 8.5) was significantly shorter than the mean first spike latency for visual stimuli $\left(58.4 \mathrm{msec}\right.$; SD 19.1), pooled variance $t_{(151)}=$ $-10.3, p<0.05$. Maximal levels of bimodal enhancement have been obtained in anesthetized preparations when the acoustic stimulus was delayed so that the initial bursts of the response to each modality overlapped in time (King and Palmer, 1985; Meredith et al., 1987), suggesting the presence of a coincidence detection mechanism to facilitate responses to stimuli located at given distances from the subject (Meredith et al., 1987). In this scheme, the faster speed of light is offset by the shorter neural latency of auditory responses.

We studied the effect of delaying the acoustic stimulus in 12 single SCi cells; two of those cells were studied at two different levels of acoustic stimulation. In addition, because the enhancement is more likely for low-level stimuli (Meredith and Stein, 1986b), the intensity of the visual stimulus used for these recordings was adjusted to render it nearly ineffective. The responses in Figure $6 A$ were recorded with an acoustic stimulus that was $20 \mathrm{~dB}$ higher than that shown in Figure $6 \mathrm{~B}$. With the more effective acoustic stimulus (Fig. $6 A$ ), the BI was $-23.5 \%$, i.e., the bimodal responses were not enhanced. The effect of delaying the acoustic stimulus by 30,50 , and $100 \mathrm{msec}$ is shown in the bottom row of Figure $6 A$. The maximal response was obtained with a delay of $100 \mathrm{msec}$, but even in this case the BI $(-12.8 \%)$ was still not enhanced. A similar pattern was observed with the lower acoustic stimulus level (Fig. $6 B$ ), but in none of the conditions did the bimodal response grossly exceed the larger of the two singlemodality responses or the sum of the single-modality responses, as seen in recordings from anesthetized preparations (Meredith and Stein, 1983, 1986b; King and Palmer, 1985). Similar results were observed in the other 11 cells studied in this same manner;
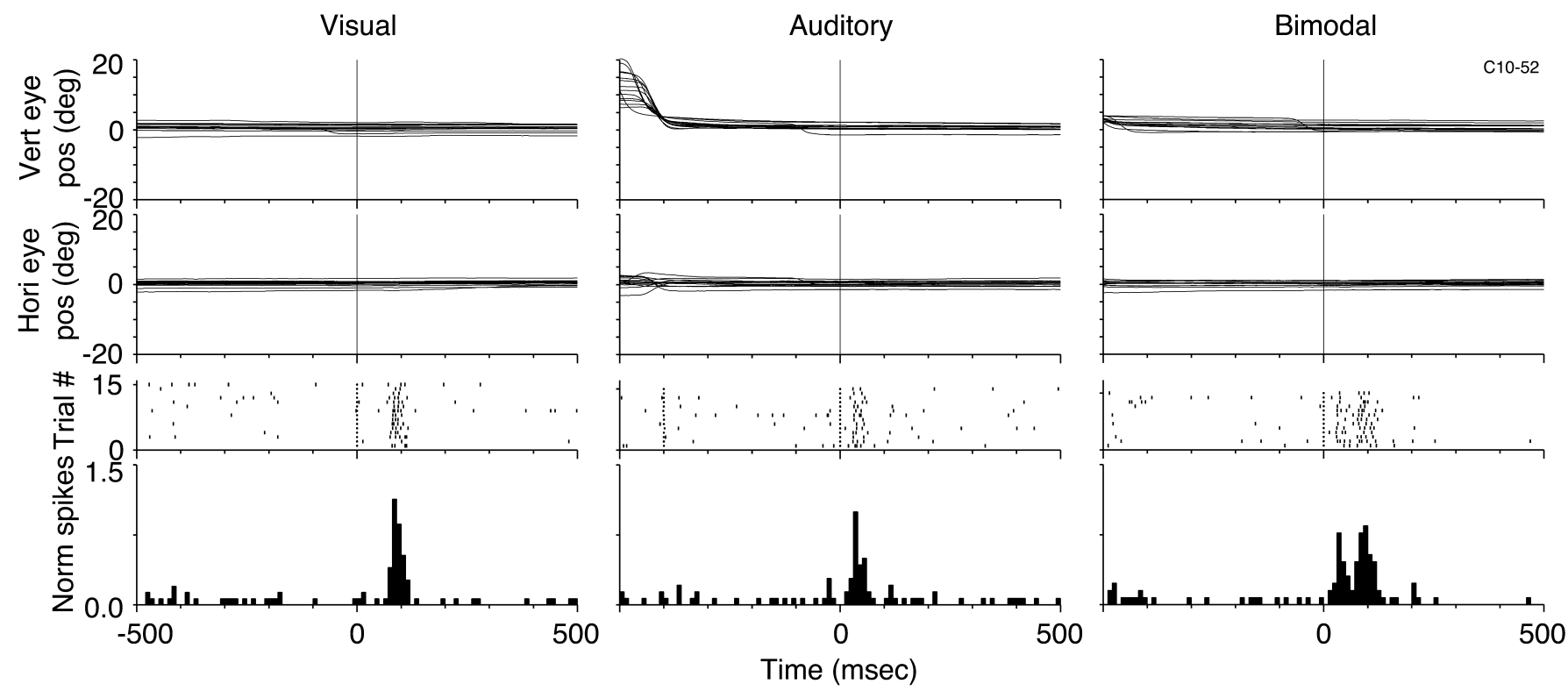

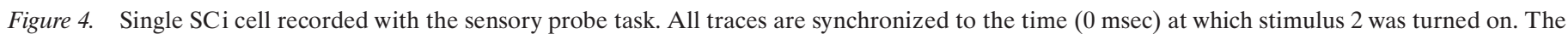

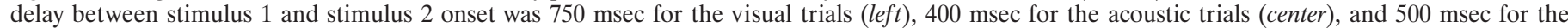

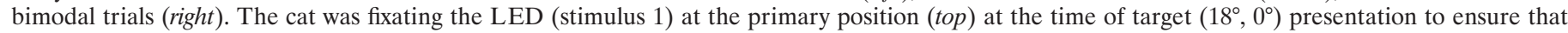

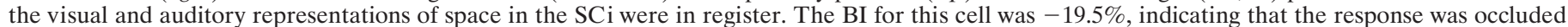

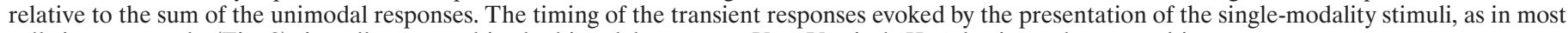
cells in our sample (Fig. 2), is well preserved in the bimodal response. Vert, Vertical; Hori, horizontal; pos, position. 


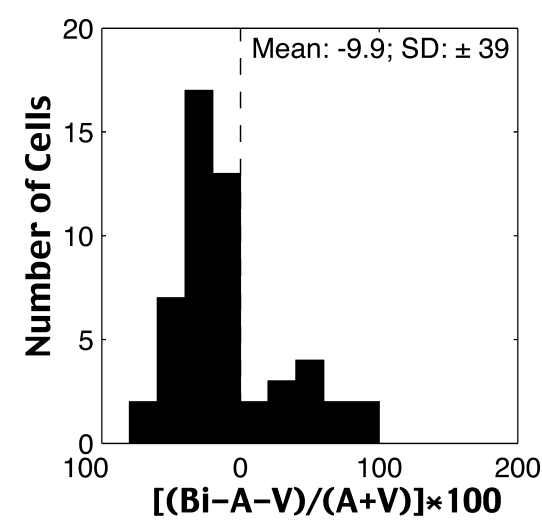

Figure 5. Summary of BI values for responses recorded with the sensory probe task as in Figure 4.

the mean BI for each of the delays was not significantly different from zero (Fig. 7). Thus, even under the most favorable conditions to evoke bimodal enhanced responses, i.e., low-intensity stimuli (Meredith and Stein, 1986b) and temporal overlap of the initial burst (King and Palmer, 1985; Meredith et al., 1987), we did not observe them in the behaving preparation.

\section{Other Bls: depression of auditory responses by visual fixation}

In addition to the bimodal suppression we found in cells with BS $<0$, we also saw other forms of suppressive BIs. The most common form was a reduction in the magnitude of single-cell responses to acoustic stimuli in the sensory probe task, in which the cat fixates a spot of light, compared with the auditory fixation task, in which no LED is on. Figure $8 A$ illustrates the responses of a single SCi cell to a $1500 \mathrm{msec}$ broadband noise burst presented through a speaker located at $\left(-18^{\circ}, 0^{\circ}\right)$ recorded with the fixation task. Like most SCi cells, this one responded to the long-duration acoustic stimulus with a burst followed by lowerfrequency activity. The responses of the same cell to an identical acoustic stimulus while fixating an LED at the primary position using the sensory probe task are shown in Figure $8 B$. The reduction in response to the same acoustic stimulus in the presence of the fixation LED is striking. The magnitude of the initial burst was reduced to approximately one-half that of the initial burst recorded in the fixation condition, and the sustained responses in Figure $8 A$ now became almost completely transient, with several single-spike trials. Figure $8 C$ illustrates the ratio of the magnitude of the responses to acoustic stimuli with LED (sensory probe trials) to without LED (fixation trials) for 62 cells from which data of this type were collected.

The depression illustrated in Figure 8 was commonly observed in our behavioral preparation. It is difficult to determine whether the depression is caused by the presence of the visual stimulus in the sensory probe trials or to the act of fixating it. More rarely, we observed cells that showed a depressive effect of the visual stimulus on the response to the acoustic stimulus independent of fixation. Figure 9 illustrates an example of an SCi cell, the auditory responses of which were completely depressed by a visual stimulus. Figure 9, top, illustrates the configuration of the stimuli used: a $1500 \mathrm{msec}$ broadband noise burst was presented at $\left(-90^{\circ}, 0^{\circ}\right)$, and a $500 \mathrm{msec}$ visual stimulus was presented during the middle of the acoustic stimulus at $\left(-18^{\circ}, 0^{\circ}\right)$. Horizontal eye position is plotted immediately below; with one exception in which the cat looked in the direction of the sound source, the eyes remained at the primary position. Figure 9, bottom, illustrates the responses of the cell. All traces are plotted synchronized to the onset of the visual stimulus at time $0 \mathrm{msec}$; the onset of the acoustic stimulus occurred at $-500 \mathrm{msec}$. The responses of the cell to the acoustic stimulus were depressed during the presentation of the visual stimulus and resumed after the visual stimulus was extinguished. Such depressive effects were rare in our sample, but they support the existence of bimodal suppression independent of visual fixation.

\section{DISCUSSION}

In anesthetized animals, the responses of single SCi cells to bimodal stimuli (auditory plus visual) grossly exceed the larger of the unimodal responses (Meredith and Stein, 1983) or the sum of the two single-modality responses (King and Palmer, 1985). Such an increase in response magnitude to bimodal stimuli has been proposed to be a mechanism to increase the likelihood of behavioral response (Stein and Meredith, 1993). This study was performed to test for the presence of bimodal enhanced responses in the SCi of behaving cats trained to orient to acoustic, visual, and bimodal targets.

To determine whether bimodal responses of SCi cells in the behaving cat were enhanced, we quantitatively evaluated their magnitude with the BI metric of King and Palmer (1985). This BI metric compares the magnitude of the bimodal response to the sum of the two single-modality responses. Meredith and Stein (1983, 1986b, 1996), on the other hand, compared the magnitude of the bimodal response to the larger of the two single-modality responses, which we have called BS, thus ignoring the contribution of the smaller of the single-modality responses. This latter metric is more suitable to evaluate the suppression of responses under bimodal stimulation and does not provide a proper measure of enhancement.

With three different behavioral tests, we found little evidence for bimodal enhancement: whereas some cells had $\mathrm{BI}>0$, the mean BI was significantly $<0$ (Fig. 3) or nearly so (Fig. 5), in sharp contrast to the large enhanced effects described in anesthetized animals (Meredith and Stein, 1983, 1986b; King and Palmer, 1985) or in the awake cat (Wallace et al., 1998). The present results are surprising because it seems reasonable to expect that bimodal enhanced responses should be just as readily observed in a behaving preparation as they are in anesthetized or alert ones (Meredith and Stein, 1983; King and Palmer, 1985; Wallace et al., 1998). The question arises, therefore, as to why we have not seen large enhanced responses in the SC of the behaving cat. We consider five possible explanations.

First, it may be that large enhanced responses are unusual and not commonly found, so they may have been missed in our sample. We do not believe this to be the case, although it is difficult to quantitatively relate our results to those presented in anesthetized animals. With the exception of Meredith et al. (1987), there are no population results in the other published studies. In addition, the metrics used to quantify BIs differ. King and Palmer (1985) reported "cross-modality" interaction indices from -95 to $452 \%$ but do not indicate the distribution or mean. In several studies of BI in the SCi of anesthetized cats, Meredith and Stein illustrate many examples of facilitation with BI of 300-1207\% (Meredith and Stein, 1986b) to as high as infinity when there were no unimodal responses, and they also do not provide population statistics or means; thus, it is difficult to ascertain how common these large enhanced responses are. The histogram in Meredith et al. (1987) showing percentage of bi- 

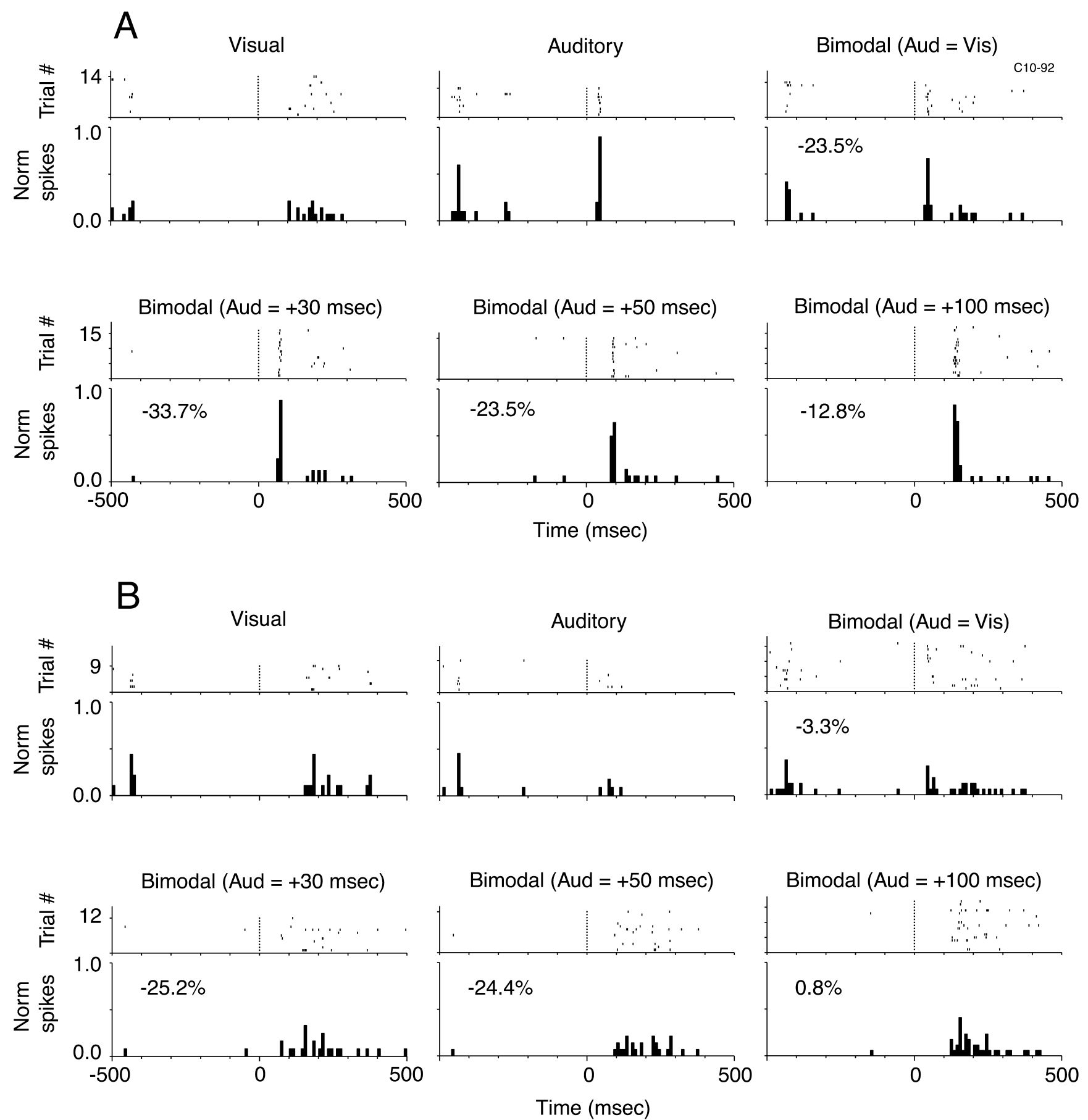

Figure 6. Single SCi bimodal cell studied with the sensory probe task at two levels of acoustic stimulation. $A$, The acoustic stimulus used in this series of recordings was $20 \mathrm{~dB}$ louder than the stimulus used in $B$. The intensity of the visual stimulus was chosen to be nearly ineffective and was maintained constant in both conditions. As in Figures 2 and 4, the responses of this cell were mostly occluded; the BI for each condition is given in each panel.

modal change, relative to the larger of the two single-modality responses, depicts two distinct populations of depressed and enhanced responses, with cases in the latter as large as $610 \%$. Judging from the available information, the impression is that large enhanced responses are common. Thus, we do not believe that we have missed an unusual response type in our sample.

Second, there is a possibility that the type of stimuli used was not appropriate for evoking enhanced responses. For example, we used stationary LEDs rather than a slowly moving bar of light as was used by Meredith and Stein (1983) or Meredith et al. (1987). Such an explanation is unlikely, however, because King and Palmer (1985) also used stationary visual stimuli and still found large bimodal enhanced responses in the SC of the anesthetized guinea pig. Furthermore, if enhanced responses are behaviorally relevant, they are unlikely to require a slowly moving bar of light. Also, it is worth noting that behavioral facilitation has been shown to occur only when the visual stimulus is delayed (Butter et al., 1989; Farah et al., 1989; Frens et al., 1995; Driver and 


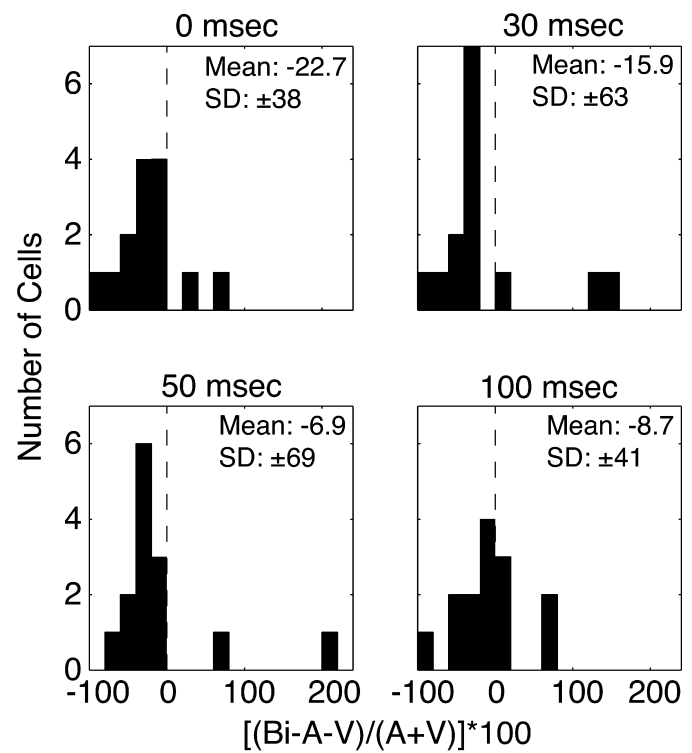

Figure 7. Summary of the BI for 12 cells studied with delayed acoustic stimuli; two cells, including that shown in Figure 6, were studied at two levels of acoustic stimulation. Each panel illustrates data from one acoustic stimulus delay $(0,30,50$, and $100 \mathrm{msec})$.

Spence, 1998), which is opposite to the configuration used by Meredith et al. (1987) and King and Palmer (1985) and to the idea that the shorter auditory latencies are offset by the faster speed of light. Similarly, saccade-related burst cells in the SCi of behaving monkeys also showed facilitation when the visual stimulus was preceded by an acoustic stimulus, but not vice versa (Frens and Van Opstal, 1998).

Third, it is possible that the intensity of the stimuli we used was too high to evoke enhanced responses (Meredith and Stein, 1986b). This explanation is also unlikely, for two reasons. First, the intensity of the visual stimulus used by King and Palmer (1985) was strong enough to evoke "vigorous discharges," as described in a previous report (King and Palmer, 1983) and by judging the magnitude and consistency of the single-modality responses shown in their paper (King and Palmer, 1985). Second, we did not observe large enhanced responses even in the recordings for which we specifically tailored our stimuli to evoke very weak responses (Figs. 6, 7). In addition, and problematically for the hypothesized functional significance of enhanced bimodal responses, Peck (1996) had to use "moderately intense" levels of stimulation to evoke orienting responses consistently.

Fourth, our animals were not trained to specifically recognize the presentation of bimodal stimuli as a special behavioral event, one that, for example, would result in a larger reward after successful completion of the behavioral task. Although modulation of this type has been documented in cortex (Platt and Glimcher, 1999), it still does not explain why enhanced interactions should be seen in anesthetized animals. Furthermore, other studies in behaving animals have also not shown enhanced sensory responses that resemble those so prominently observed in anesthetized preparations (Peck, 1996; Frens and Van Opstal, 1998). Conversely, Wallace et al. (1998) reported prominent enhanced responses in the SCi of the alert, nonbehaving cat. We can offer no reasonable explanation for the discrepancies in our results except to point out that their short communication showed results from only two cells, again without any population data on the degree to which BIs differed from additive. Their measurements of response latencies of auditory and visual responses, on the other hand, are similar to ours, with longer mean visual latency ( $80.8 \mathrm{msec}$ vs $58.4 \mathrm{msec}$ in our responses) and virtually identical auditory latencies (17.6 $\mathrm{msec}$ vs $17.7 \mathrm{msec}$ in ours).

Fifth, the SC is endowed with both a vast intrinsic inhibitory network (Mize et al., 1994) and numerous inhibitory inputs
Without LED

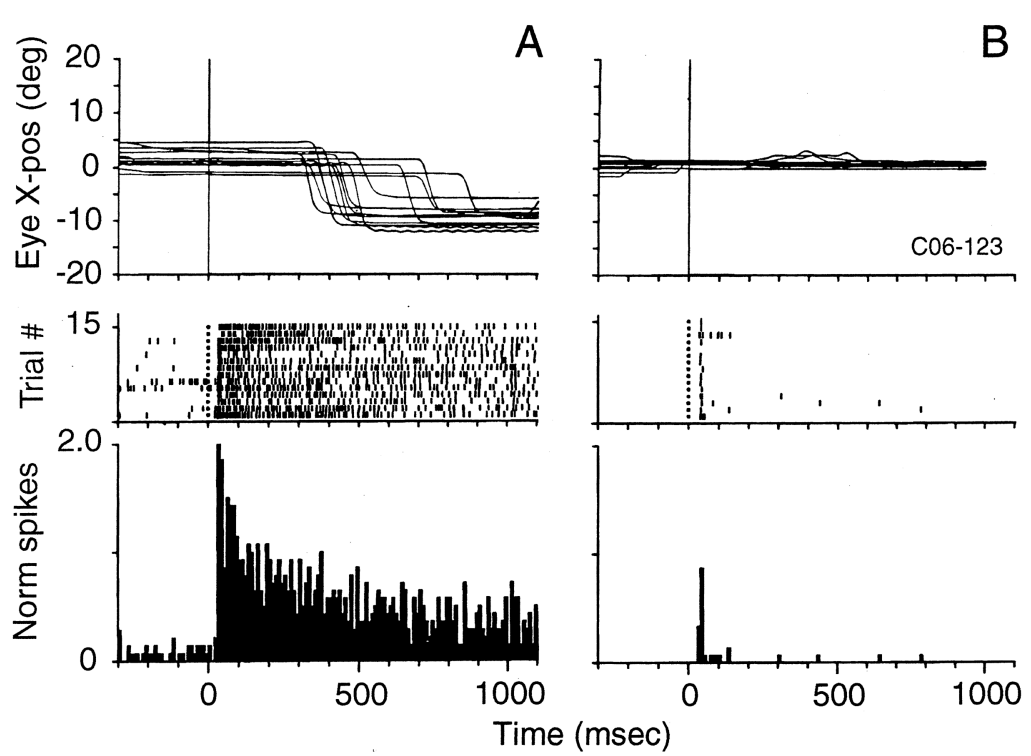

Summary

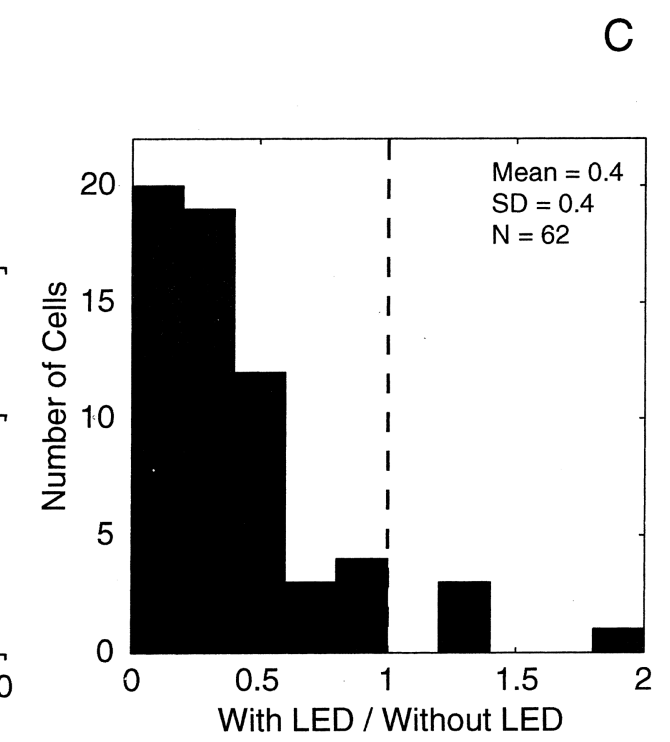

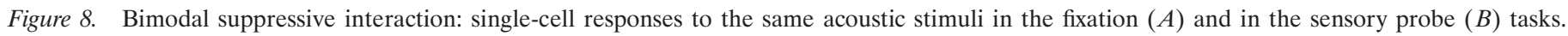

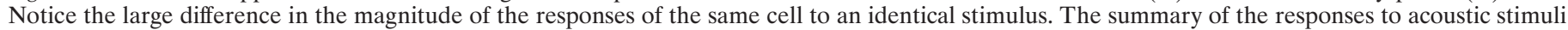
from 62 cells, expressed as the ratio of response magnitude with LED to that recorded without LED, is shown in $C$. 


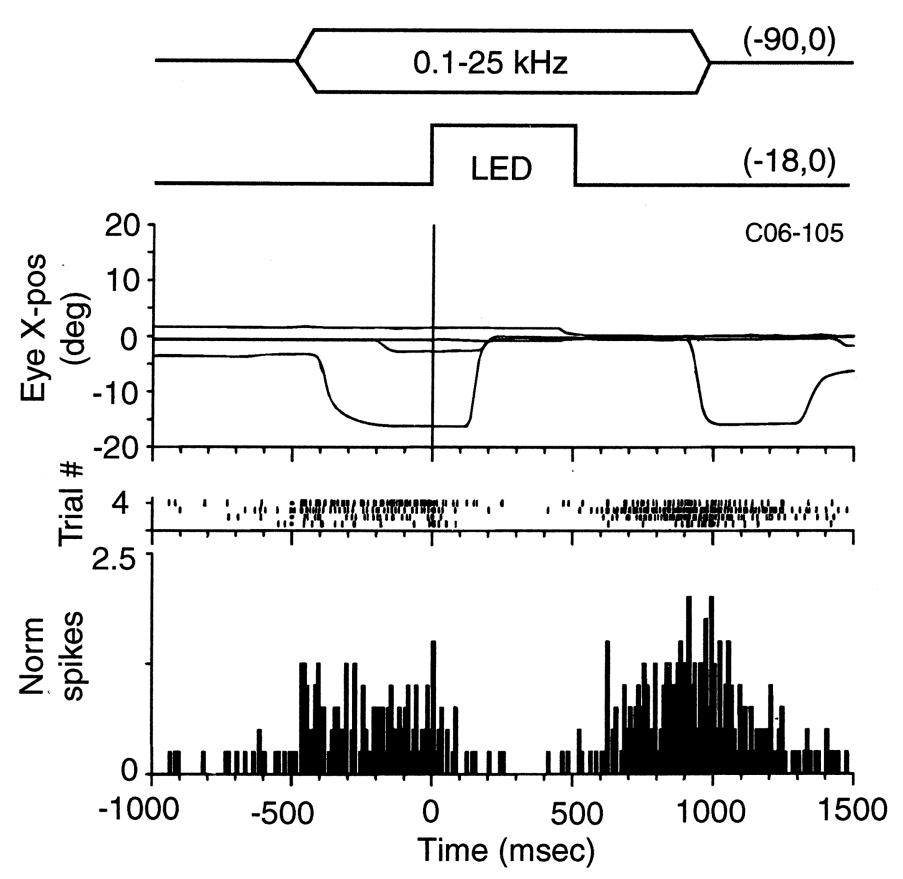

Figure 9. Bimodal inhibitory interaction. The stimuli configuration used for these recordings is illustrated at the top. The acoustic stimulus was located at $\left(-90^{\circ}, 0^{\circ}\right)$; the visual one at $\left(-18^{\circ}, 0^{\circ}\right)$. Other details are as in Figure 2. The responses of a single SCi cell to acoustic stimuli, which were atypically sustained, were suppressed by the presentation of a visual stimulus. Notice that the responses to the acoustic stimulus resumed after the visual stimulus was turned off.

(Appell and Behan, 1990); prominent among the latter is a GABAergic input from the substantia nigra pars reticulata (Graybiel, 1978; Harting et al., 1988), the integrity of which is required for normal oculomotor function (Boussaoud and Joseph, 1985; Hikosaka and Wurtz, 1985). It is therefore possible that anesthetics interfere with the normal balance between excitation and inhibition in the SC and/or some of its inputs, which results in enhanced bimodal responses.

On the other hand, the depression of auditory responses by fixation of a visual stimulus was commonly observed. In fact, in many cases the effect was so strong that it practically precluded us from using the sensory probe task because it silenced the responses of the cells to the stimulus of interest. Similar interactions, although between two visual stimuli, have been reported by Rizzolatti et al. (1974) in the SC of the awake, paralyzed cat and by Weyand and Gafka (1998) in area 6 of the behaving cat's cortex. There seems to be no obvious functional significance for such strong depression, but it has been suggested that it might be a manifestation of mechanisms underlying the animal's allocation of attention to stimuli in the visual field (Rizzolatti et al., 1974). In such a context, our observations point to a more general attentional mechanism that involves stimuli of different modalities, not just vision.

\section{REFERENCES}

Appell PP, Behan M (1990) Sources of subcortical GABAergic projections to the superior colliculus in the cat. J Comp Neurol 302:143-158.

Boussaoud D, Joseph JP (1985) Role of the cat substantia nigra pars reticulata in eye and head movements. II. Effects of local pharmacological injections. Exp Brain Res 57:297-304.

Butter CM, Buchtel HA, Santucci R (1989) Spatial attentional shifts: Further evidence for the role of polysensory mechanisms using visual and tactile stimuli. Neuropsychologia 27:1231-1240.
Cynader M, Berman N (1972) Receptive-field organization of monkey superior colliculus. J Neurophysiol 35:187-201.

Driver J, Spence C (1998) Attention and the crossmodal construction of space. Trends Cogn Sci 2:254-262.

Farah MJ, Wong AB, Monheit MA, Morrow LA (1989) Parietal lobe mechanisms of spatial attention: modality-specific or supramodal? Neuropsychologia 27:461-470.

Finlay BL, Schneps SE, Wilson KG, Schneider GE (1978) Topography of visual and somatosensory projections to the superior colliculus of the golden hamster. Brain Res 142:223-235.

Frens MA, Van Opstal AJ (1998) Visual-auditory interactions modulate saccade-related activity in monkey superior colliculus. Brain Res Bull 46:211-224.

Frens MA, Van Opstal AJ, Van der Willigen RF (1995) Spatial and temporal factors determine auditory-visual interactions in human saccadic eye movements. Percept Psychophys 57:802-816.

Gordon B (1973) Receptive fields in deep layers of the cat superior colliculus. J Neurophysiol 36:157-178.

Graybiel AM (1978) Organization of the nigrotectal connection: an experimental tracer study in the cat. Brain Res 143:339-348.

Guitton D, Crommelinck M, Roucoux A (1980) Stimulation of the superior colliculus in the alert cat. I. Eye movements and neck EMG activity evoked when the head is restrained. Exp Brain Res 39:63-73.

Harting JK, Huerta MF, Hashikawa T, Weber JT, Van Lieshout DP (1988) Neuroanatomical studies of the nigrotectal projection in the cat. J Comp Neurol 278:615-631.

Hikosaka O, Wurtz RH (1985) Modification of saccadic eye movements by GABA-related substances. II. Effects of muscimol in monkey substantia nigra pars reticulata. J Neurophysiol 53:292-308.

Hirsch JA, Chan JCK, Yin TCT (1985) Responses of neurons in the cat's superior colliculus to acoustic stimuli. I. Monaural and binaural response properties. J Neurophysiol 53:726-745.

Huerta MF, Harting JK (1984) The mammalian superior colliculus: studies of its morphology and connections. In: Comparative neurology of the optic tectum (Vanegas H, ed), pp 687-773. New York: Plenum.

Jay MF, Sparks DL (1987) Sensorimotor integration in the primate superior colliculus. II. Coordinates of auditory signals. J Neurophysiol 57:35-55.

Judge SJ, Richmond BJ, Chu FC (1980) Implantation of magnetic search coils for measurement of eye position: an improved method. Vision Res 20:535-538.

King AJ, Palmer AR (1983) Cells responsive to free-field auditory stimuli in guinea-pig superior colliculus: distribution and response properties. J Physiol (Lond) 342:361-381.

King AJ, Palmer AR (1985) Integration of visual and auditory information in bimodal neurones in the guinea-pig superior colliculus. Exp Brain Res 60:492-500.

Meredith MA, Stein BE (1983) Interactions among converging sensory inputs in the superior colliculus. Science 221:389-391.

Meredith MA, Stein BE (1985) Descending efferents from the superior colliculus relay integrated multisensory information. Science 227:657-659.

Meredith MA, Stein BE (1986a) Spatial factors determine the activity of multisensory neurons in cat superior colliculus. Brain Res 365:350-354.

Meredith MA, Stein BE (1986b) Visual, auditory, and somatosensory convergence on cells in superior colliculus results in multisensory integration. J Neurophysiol 56:640-662.

Meredith MA, Stein BE (1996) Spatial determinants of multisensory integration in cat superior colliculus neurons. J Neurophysiol 75:1843-1857.

Meredith MA, Nemitz JW, Stein BE (1987) Determinants of multisensory integration in superior colliculus neurons. I. Temporal factors. J Neurosci 7:3215-3229.

Meredith MA, Clemo HR, Stein BE (1991) Somatotopic component of the multisensory map in the deep laminae of the cat superior colliculus. J Comp Neurol 312:353-370.

Middlebrooks LC, Knudsen EI (1984) A neural code for auditory space in the cat's superior colliculus. J Neurosci 4:2621-2634.

Mize RR, Whitworth H, Nunes-Cardozo B, van der Want J (1994) Ultrastructural organization of GABA in the rabbit superior colliculus revealed by quantitative postembedding immunocytochemistry. J Comp Neurol 341:273-287.

Newman EA, Hartline PH (1981) Integration of visual and infrared information in bimodal neurons in the rattlesnake optic tectum. Science 213:789-791.

Peck CK (1996) Visual-auditory integration in cat superior colliculus: implications for neuronal control of the orienting response. In: Progress in brain research, Vol 112, Chap 12 (Norita M, Bando TB, Stein B, eds), pp 167-177. Amsterdam: Elsevier.

Platt ML, Glimcher PW (1999) Neural correlates of decision variables in parietal cortex. Nature 400:233-238.

Populin LC, Yin TCT (1997) Auditory-visual integration in the superior colliculus of the behaving cat. Soc Neurosci Abstr 24:1295.

Populin LC, Yin TCT (1998a) Sensitivity of auditory cells in the superior colliculus to eye position in the behaving cat. In: Psychological and 
physiological advances in hearing (Palmer AR, Summerfield AQ, Meddis R, eds), pp 441-448. London: Whurr.

Populin LC, Yin TCT (1998b) Behavioral studies of sound localization in the cat. J Neurosci 18:2147-2160.

Rizzolatti G, Camarda R, Grupp LA, Pisa M (1974) Inhibitory effect of remote visual stimuli on visual responses of cat superior colliculus: spatial and temporal factors. J Neurophysiol 37:1262-1275.

Robinson DA (1963) A method of measuring eye movement using a scleral search coil in a magnetic field. IEEE Trans Biomed Electron BME 10:137-145.

Robinson DA (1972) Eye movements evoked by collicular stimulation in the alert monkey. Vision Res 12:1795-1808.

Schiller PH, Stryker M (1972) Single-unit recording and stimulation in superior colliculus of the alert rhesus monkey. J Neurophysiol 35:915-924.

Schröger E, Widman A (1998) Speeded responses to audiovisual signal changes result from bimodal integration. Psychophysiology 35:755-759.

Stein BE, Clamann HP (1981) Control of pinna movements and senso- rimotor register in cat superior colliculus. Brain Behav Evol 19:180-192.

Stein BE, Meredith MA (1993) The merging of the senses. Cambridge, MA: MIT

Stein BE, Magalhaes-Castro B, Kruger L (1976) Relationship between visual and tactile representations in cat superior colliculus. J Neurophysiol 39:401-419.

Stein BE, Meredith MA, Huneycutt WS, McDade L (1989) Behavioral indices of multisensory integration: orientation to visual cues is affected by auditory stimuli. J Cognit Neurosci 1:12-24.

Taylor TL, Klein RM, Munoz DP (1999) Saccadic performance as a function of the presence and disappearance of auditory and visual fixation stimuli. J Cognit Neurosci 11:206-213.

Wallace MT, Meredith MA, Stein BE (1998) Multisensory integration in the superior colliculus of the alert cat. J Neurophysiol 80:1006-1010.

Weyand TG, Gafka AC (1998) Activity of neurons in area 6 of the cat during fixation and eye movements. Vis Neurosci 15:123-140.

Wise LZ, Irvine DRF (1983) Auditory response properties of neurons in deep layers of cat superior colliculus. J Neurophysiol 49:674-685. 